\title{
The Relationship Between Serum 25-Hydroxyvitamin D Levels and Physical Performance in Community-Dwelling Older Adults
}

\author{
Hana Moon ${ }^{1}$, Hae-Jin $\mathrm{Ko}^{2,3}$, A-Sol Kim ${ }^{1,2}$ \\ ${ }^{1}$ Department of Family Medicine, Kyungpook National University Chilgok Hospital, Daegu, Korea \\ ${ }^{2}$ Department of Family Medicine, School of Medicine, Kyungpook National University, Daegu, Korea \\ ${ }^{3}$ Department of Family Medicine, Kyungpook National University Hospital, Daegu, Korea
}

Corresponding Author:

Hae-Jin Ko, MD, PhD

https://orcid.org/0000-0003-4460-1476

Department of Family Medicine,

Kyungpook National University

Hospital, 130 Dongdeok-ro, Jung-gu,

Daegu 41944, Korea

E-mail: liveforme@knu.ac.kr

Received: January 6, 2019

Revised: March 5, 2019

Accepted: March 7, 2019

\begin{abstract}
Background: Vitamin D deficiency is a risk factor for musculoskeletal health in older adults. While many studies have explored the relationship between vitamin $D$ deficiency and fractures, few have examined the relationship between vitamin $\mathrm{D}$ and physical performance. We, therefore, sought to evaluate the relationship between serum 25-hydroxyvitamin D $[25(\mathrm{OH}) \mathrm{D}]$ levels and physical performance in community-dwelling older adults. Methods: A single-center, cross-sectional study was performed using data collected from 132 individuals aged 60 years or older who were living independently and who participated in the National Health Insurance Service health check-up between May and December 2016. Physical performance was assessed using a short physical performance battery (SPPB). Linear regression was used to examine the association between 25(OH)D levels and physical performance after adjustment for sociodemographic variables, behavioral characteristics, and body mass index. Results: Approximately $36.5 \%$ of male and $50.7 \%$ of females had vitamin D levels indicative of deficiency (serum $25(\mathrm{OH}) \mathrm{D}<20.0 \mathrm{ng} / \mathrm{mL}$ ). $25(\mathrm{OH}) \mathrm{D}$ levels were significantly associated with the chair stand SPPB scores in male and females but not with those of the walking and balance tests. After adjustment for potential confounders, there remained a linear association between 25(OH)D levels and the chair stand test for both sexes. Conclusion: Lower serum 25(OH)D levels were associated with poor physical performance (chair stand score) among community-dwelling older adults in Korea. Further prospective studies are needed to verify these results. (Ann Geriatr Med Res 2019;23:9-15)
\end{abstract}

Key Words: Vitamin D, Physical functional performance, Cross-sectional study, Aged

\section{INTRODUCTION}

Vitamin D plays an important role in bone and mineral metabolism. Severe vitamin D deficiency leads to bone diseases such as rickets in children and osteomalacia in adults. ${ }^{1)}$ Humans acquire vitamin D in two ways: from the diet and via cutaneous synthesis driven by sunlight. The active form of vitamin D is a potent steroid hormone that promotes the proliferation and differentiation of muscle cells via vitamin D receptors (VDRs) in skeletal muscle. ${ }^{2-4)}$ Therefore, inadequate vitamin D can lead to the disturbance of muscle protein synthesis and reduced muscle mass and strength. ${ }^{5-8)}$ The increased secretion of parathyroid hormone in vitamin D insufficiency (secondary hyperparathyroidism) contributes to bone fragility, fractures, and impaired muscle function. ${ }^{1,9,10)}$

Vitamin D deficiency has recently attracted increasing interest among geriatric clinicians because the condition is common in older adults and can result in increased susceptibility to falls and osteoporotic fractures later in life. ${ }^{6,11)}$ In a recent Korean study, the prevalence of inadequate vitamin $\mathrm{D}$ levels was high among older urban populations, at $63.6 \%$ in males and $63.7 \%$ in females. ${ }^{12)}$ In Korea, where a majority of the population lives in the cities, senior citizens spend significant time indoors and have insufficient ultraviolet B exposure for adequate cutaneous vitamin D production. Even when they go outdoors, older adults tend to apply sunscreen and avoid wearing shortsleeved shirts or short shorts. Furthermore, the capacity to synthesize vitamin D in the skin decreases with age. ${ }^{13)}$ The number of VDRs in skeletal muscle also decreases with age, which can lead to a decline in muscle strength in older populations. ${ }^{14)}$

Although the effect of vitamin D in maintaining musculoskeletal health is well established, the understanding of how vitamin D affects physical performance remains limited. ${ }^{6,11,14-18)}$ Previous studies have explored the relationship between vitamin D deficiency and fracture but relatively 
few have focused on the relationship between vitamin D and physical performance in Asian populations, especially in Korean older populations. ${ }^{19)}$ Therefore, the aim of this study was to examine the association between vitamin D levels and physical performance among communitydwelling older adults in Korea.

\section{MATERIALS AND METHODS}

\section{Study Population}

This single-center, cross-sectional study was conducted using data collected from 165 individuals aged 60 years or older who participated in the National Health Insurance Service health check-ups between May 10, 2016, and December 8, 2016. The inclusion criteria were as follows: (1) age 60 years or over; (2) able to walk $10 \mathrm{~m}$ independently with or without a walking aid; and (3) living independently and able to go outside on a regular basis. The exclusion criteria were as follows: (1) withdrawal of consent; (2) incomplete data; (3) prolonged glucocorticoid use (more than 3 months at any time); (4) hormone replacement therapy; (5) use of calcium or vitamin D as a nutritional supplement; (6) use of osteoporosis medication or advanced renal failure (estimated glomerular filtration rate $<30 \mathrm{~mL} / \mathrm{min}$ ); (7) cognitive impairments limiting the ability to understand the motor tasks required during testing; (8) central nervous system disorders, peripheral nervous system disorders, or myopathies; (9) previous lower extremity surgery, and (10) current or past cancer.

All procedures performed in studies involving human participants were in accordance with the ethical standards of the institutional and/or national research committee and with the 1964 Declaration of Helsinki and its later amendments or comparable ethical standards. Ethical approval was provided by the Institutional Review Board of Kyungpook National University Hospital (IRB protocol number KNUH 2016-04-016-011). All participants were informed about the study and provided written consent to participate.

\section{Demographic and Clinical Characteristics}

Height, weight, and waist circumference were measured by a trained nurse. Waist circumference was measured as the smallest circumference at or below the costal margin, while hip circumference was measured at the level of the greater trochanter using a fiberglass tape measure at a tension of $600 \mathrm{~g}$. Body mass index (BMI) was calculated as weight in kilograms divided by height in square meters $(\mathrm{kg} /$ $\mathrm{m}^{2}$ ). Obesity was defined as a BMI $\geq 25 \mathrm{~kg} / \mathrm{m}^{2}{ }^{20)}$ After 5 minutes of rest, blood pressure was measured twice using automatic oscillometric devices (BPBIO320; InBody, Seoul, Korea) and the mean value ( $\mathrm{mmHg}$ ) was used as the final measurement.

To collect detailed information regarding smoking status, alcohol consumption, and physical activity, standardized questionnaires were administered by trained health professionals. Cigarette smoking was assessed using two questions validated by the Centers for Disease Control and Prevention Behavioral Risk Factor Surveillance System: "Have you smoked at least 100 cigarettes in your entire life?" and "Do you now smoke cigarettes every day, some days, or not at all?" The responses were grouped into two categories: never smoker and ever smoker. Ever smokers were defined as those persons who reported smoking at least 100 cigarettes in their lifetime. Never smokers were defined as persons who reported never having smoked 100 cigarettes. ${ }^{21)}$ Self-reported alcohol consumption was assessed as the number of drinks/week (one drink=14 $\mathrm{g}$ of alcohol). Based on their responses, the participants were categorized as social or binge drinkers. As per the Center for Disease Control and Prevention criteria, binge drinking was defined as the consumption of more than five and four (for males and females, respectively) consecutive drinks of any type of alcohol within the past 12 months. ${ }^{22}$ Participants were asked a binary (yes/no) question related to physical activity. People who reported moderate physical activity (3-6 metabolic equivalents) for at least 150 minutes per week were defined as having "good physical activity"; the remainder were defined as having "poor physical activity". ${ }^{23}$

Comorbidity was defined as being diagnosed with three or more of the following diseases: stroke, heart disease (myocardial infarction or angina), hypertension, diabetes mellitus, dyslipidemia, or pulmonary tuberculosis.

\section{Measurement of Vitamin D and Other Laboratory Tests}

After fasting for at least 8 hours, all participants visited the health-promotion centers on the morning of the appointed day. Serum was isolated by centrifugation (F5220; Kubota Corporation, Saitama, Japan) within 1 hour of collection and kept refrigerated $\left(2^{\circ} \mathrm{C}-8^{\circ} \mathrm{C}\right)$ until use. The serum concentration of 25(OH)D was used as a measure of vitamin D status. Levels of 25(OH)D were measured by radioimmunoassay using a Packard COBRA Gamma Counter (DiaSorin; Saluggia, Vercelli, Italy). The reference range for the assay is $8.9-37.6 \mathrm{ng} / \mathrm{mL}$, with intra- and inter-assay coefficients of variation of $8.1 \%$ and $10.2 \%$, respectively. Vitamin D deficiency was defined as a 25(OH)D level $<20$ $\mathrm{ng} / \mathrm{mL}$, and the definitions of insufficiency was defined as a 25(OH)D level $<30 \mathrm{ng} / \mathrm{mL}^{24)}$ The following were measured in all participants: markers of liver function (alanine aminotransferase and aspartate aminotransferase); lipid profiles (total cholesterol, low-density lipoprotein, highdensity lipoprotein, and triglycerides); complete blood count; blood urea nitrogen; serum creatinine; and fasting blood glucose. Serum creatinine was measured by a Beckman Coulter analyzer (AU 2700; Beckman Coulter Diagnostics, Brea, CA, USA) using Jaffe's method. Calibration was performed using isotope dilution mass spectroscopy. Serum total cholesterol, low-density lipoprotein, highdensity lipoprotein, triglycerides, and glucose, as well as the other basic biochemical blood parameters, were mea- 
sured using standard chemical and enzymatic commercial methods on a Hitachi analyzer (D-2400; Hitachi HighTechnologies Corporation, Tokyo, Japan).

\section{Assessment of Physical Performance}

A short physical performance battery (SPPB) was used to assess physical performance. The SPPB test is an objective assessment tool for the evaluation of lower extremity function in older adults. It was developed by the National Institute of Aging and is used widely in the geriatric literature. ${ }^{25)}$ The SPPB includes a 3-meter walking test, a chair stand test (standing up from a chair as quickly as possible without using hands (repeated five times), and a balance test (standing with feet in "tandem stand" for up to 30 seconds). Participants score $0-4$ points for each test, with a maximum of 12 points and higher scores indicating better performance. Scores of 0-3, 4-6, 7-9, and 10-12 are classified as severe, moderate, mild, and minimal lower extremity limitations, respectively. ${ }^{26)}$

\section{Statistical Analyses}

Statistical calculations were performed using IBM SPSS statistics for Windows, version 21.0 (IBM Co., Armonk, NY, USA). P-values of less than 0.05 were considered statistically significant. T-tests or Kruskal-Wallis tests were used to compare continuous variables, while Pearson's chi-square tests were used to compare categorical variables. The summarized statistics are presented as means \pm standard deviation (continuous variables) and as numbers (percentage) (categorical variables). The participants were divided into quartiles according to their $25(\mathrm{OH})$ D level. Since serum vitamin D levels are sex-dependent, males and female older adults were subjected to stratified analyses. ${ }^{27-29)}$ Separate analyses were performed for each physical function outcome test (3-meter walking test, chair stand test, and balance test). In Model 1, linear regression analysis was performed with serum 25(OH)D as an independent variable and each SPPB score as a dependent variable. The model was adjusted for age, smoking status, physical activity, and BMI (all factors that might affect physical performance). ${ }^{30-32)}$ Model 2 was adjusted for age. Model 3 was adjusted for the variables used in Model 2 plus smoking status, alcohol consumption, physical activity, BMI, and comorbidities. All independent variables were included in the regression model simultaneously using the enter method.

Table 1. Subject characteristics

\begin{tabular}{|c|c|c|c|c|}
\hline \multicolumn{2}{|c|}{ Characteristic } & Males $(n=63)$ & Females $(\mathrm{n}=69)$ & p-value* \\
\hline \multicolumn{2}{|l|}{ Age (y) } & $68.30 \pm 6.14$ & $68.45 \pm 5.87$ & 0.888 \\
\hline \multicolumn{2}{|c|}{ Serum 25(OH)D (ng/mL) } & $23.61 \pm 9.07$ & $22.55 \pm 11.32$ & 0.556 \\
\hline \multicolumn{2}{|c|}{ Prevalence of vitamin D deficiency } & $23(36.5)$ & $35(50.7)$ & \\
\hline \multicolumn{5}{|l|}{ SPPB score } \\
\hline \multicolumn{2}{|l|}{ Balance } & $4.00 \pm 0.00$ & $3.96 \pm 0.27$ & 0.182 \\
\hline \multicolumn{2}{|l|}{ Walking } & $3.90 \pm 0.43$ & $3.90 \pm 0.46$ & 0.936 \\
\hline \multicolumn{2}{|l|}{ Chair } & $3.65 \pm 0.72$ & $3.62 \pm 0.84$ & 0.840 \\
\hline \multicolumn{2}{|l|}{ Total } & $11.57 \pm 1.04$ & $11.48 \pm 1.24$ & 0.641 \\
\hline \multirow[t]{2}{*}{ Smoking } & Never & $55(87.3)$ & $68(98.6)$ & $<0.001^{\dagger}$ \\
\hline & Ever & 8 (12.7) & $1(1.4)$ & \\
\hline \multirow[t]{2}{*}{ Drinking } & Social & $56(88.8)$ & $69(100.0)$ & $0.004^{\dagger}$ \\
\hline & Binge & $7(11.1)$ & $0(0.0)$ & \\
\hline \multirow[t]{2}{*}{ Physical activity } & Good & $19(30.2)$ & $13(18.8)$ & 0.095 \\
\hline & Poor & $44(69.8)$ & $56(81.2)$ & \\
\hline \multirow[t]{2}{*}{ Obesity } & Normal & $37(58.7)$ & $50(72.5)$ & 0.070 \\
\hline & Obese & $26(41.3)$ & $19(27.5)$ & \\
\hline \multirow[t]{2}{*}{ Comorbidity } & $<3$ & $53(84.1)$ & $62(89.9)$ & 0.235 \\
\hline & $\geq 3$ & $10(15.9)$ & $7(10.1)$ & \\
\hline
\end{tabular}

Values are expressed as mean \pm standard deviation or number (\%).

SPPB, short physical performance battery; 25(OH)D, serum 25-hydroxyvitamin D level.

*Independent t-tests were used to compare continuous variables, while Pearson's chi-square tests were used to compare categorical variables. "Fisher's exact test.

Vitamin D deficiency was defined as a 25(OH)D level $<20 \mathrm{ng} / \mathrm{mL}$. Ever smokers were defined as persons who reported smoking at least 100 cigarettes in their lifetime; the remaining participants were defined as never smokers. Binge drinking was defined as the consumption of more than five and four drinks of any alcohol in a row over the past 12 months for males and females, respectively. Individuals who reported moderate physical activity (3-6 metabolic equivalents) for at least 150 minutes per week were defined as having good physical activity; the remaining participants were defined as having poor physical activity. Obesity was defined as a body mass index of $\geq 25 \mathrm{~kg} / \mathrm{m}^{2}$. Comorbidity was defined as having three or more diagnosed diseases among stroke, heart disease (myocardial infarction or angina), hypertension, diabetes mellites, dyslipidemia, pulmonary tuberculosis, and malignancy. 


\section{RESULTS}

\section{Subject Characteristics}

Overall, 165 participants who met the inclusion criteria were recruited; 33 participants who withdrew their consent were excluded. Complete data were available for 132 (63 males, 47.7\%) participants who were enrolled in the final analysis. All participants were Korean. The sex-specific characteristics of the study population are shown in Table 1. The mean ages of the males and females in the study were 68.30 (range, 60-85) and 68.45 (range, 60-84) years, respectively.

Obesity was more common in males than in females (41.3\% vs. $27.5 \%$; $p=0.070)$. Significantly fewer females than males were ever smokers $(1.4 \%$ vs. $12.7 \%$; $p<0.001)$. Binge drinking was reported by $11.1 \%$ of males and by no females ( $p=0.004)$. In addition, $30.2 \%$ of males and $18.8 \%$ of females were physically active and $15.9 \%$ of males and $10.1 \%$ of females had comorbidities.

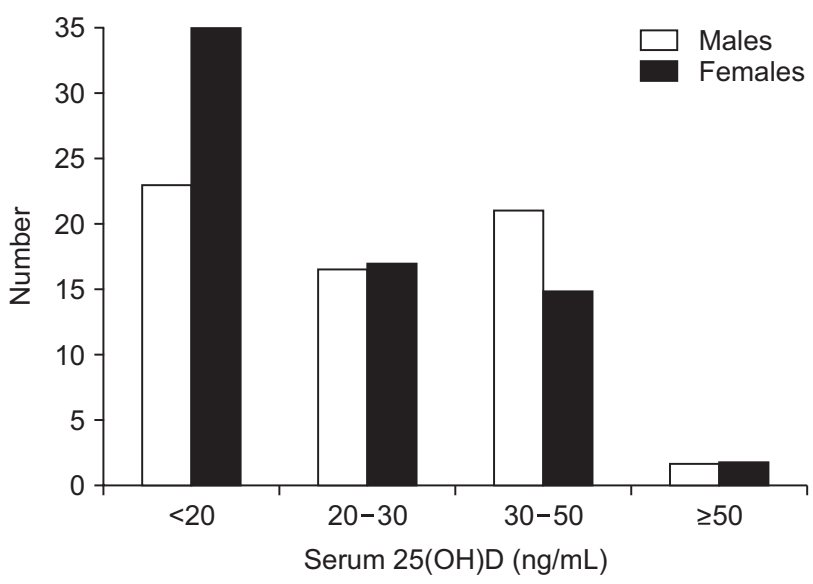

Fig. 1. Distribution of 25-hydroxyvitamin D [25(OH)D] levels of the subject. Distribution of 25(OH)D levels in males and females.

\section{Vitamin D Levels}

Fig. 1 shows the distribution of 25(OH)D levels. Vitamin D deficiency (serum 25(OH)D, <20.0 ng/mL) was highly prevalent in this population. More females than males were deficient (36.5\% and 50.7\%, respectively). There were no significant differences in mean serum 25(OH)D levels between males $(23.61 \pm 9.07 \mathrm{ng} / \mathrm{mL})$ and females $(22.55 \pm 11.33$ $\mathrm{ng} / \mathrm{mL})(\mathrm{p}=0.556)$.

\section{Physical Performance According to Vitamin D Quartile}

The results of the physical performance tests according to serum 25(OH)D quartile are shown in Table 2. For females, 25(OH)D status was positively associated with better chair stand test scores ( $p$ for trend $=0.018$ ) but there were no significant differences among the 25(OH)D quartiles with respect to the other SPPB scores. For males, the $25(\mathrm{OH})$ D status across all four quartiles was positively associated with balance scores and there were no significant differences among 25(OH)D quartiles with respect to the other SPPB scores.

\section{Associations Between Vitamin D Levels and Physical Performance}

Model 1 revealed that lower 25(OH)D levels in females were associated with worse chair stand test scores $(\beta=0.213$, $\mathrm{p}=0.018$ ), independent of age, BMI, comorbidity, and lifestyle factors (alcohol consumption, smoking, and physical activity) ( $\beta=0.176, p=0.046$; Model 3). However, there were no associations between 25(OH)D levels and balance test and walking test scores $(\mathrm{p}=0.626$ and $\mathrm{p}=0.615$, respectively; Model 3).

For males, Models 1 and 2 revealed no significant association between 25(OH)D levels and any SPPB score. However, in the fully-adjusted model (Model 3), lower 25(OH) $\mathrm{D}$ levels were significantly associated with a worse chair stand score $(\beta=-0.168, p=0.036)$ (Table 3$)$.

Table 2. Physical performance scores according to 25(OH)D quartiles

\begin{tabular}{|ccccccc}
\hline \multicolumn{1}{c}{ Variable } & $\mathrm{Q} 1$ & $\mathrm{Q} 2$ & $\mathrm{Q} 3$ & $\mathrm{Q} 4$ & $\mathrm{p}$-value & $\mathrm{p}$ for trend \\
\hline 25(OH)D quartile in males & 15 & 16 & 16 & 16 & \\
Range of 25(OH)D $(\mathrm{ng} / \mathrm{mL})$ & $9.28-16.53$ & $16.54-22.57$ & $22.58-28.60$ & $28.61-51.19$ & \\
\hline Balance & $4.00 \pm 0.00$ & $4.00 \pm 0.00$ & $4.00 \pm 0.00$ & $4.00 \pm 0.00$ & 1.000 & $\mathrm{NA}$ \\
Walking & $4.00 \pm 0.00$ & $3.88 \pm 0.50$ & $3.88 \pm 0.50$ & $3.88 \pm 0.50$ & 0.809 & 0.455 \\
\hline Chair & $3.87 \pm 0.35$ & $3.69 \pm 0.60$ & $3.63 \pm 0.72$ & $3.44 \pm 1.03$ & 0.733 & 0.100 \\
Total SPPB & $11.93 \pm 0.26$ & $11.56 \pm 1.03$ & $11.50 \pm 1.10$ & $11.31 \pm 1.40$ & 0.462 & 0.107 \\
25(OH)D quartile in females & 17 & 17 & 18 & 17 & \\
Range of 25(OH)D $(\mathrm{ng} / \mathrm{mL})$ & $9.19-14.16$ & $14.17-19.47$ & $19.48-30.35$ & $30.36-64.13$ & \\
Balance & $3.88 \pm 0.49$ & $4.00 \pm 0.00$ & $4.00 \pm 0.00$ & $3.94 \pm 0.00$ & 0.554 & 0.542 \\
Walking & $3.76 \pm 0.75$ & $3.94 \pm 0.24$ & $4.00 \pm 0.32$ & $3.88 \pm 0.49$ & 0.529 & 0.406 \\
\hline Chair & $3.24 \pm 1.15$ & $3.53 \pm 0.87$ & $3.89 \pm 0.32$ & $3.82 \pm 0.73$ & 0.089 & 0.018 \\
Total SPPB & $10.88 \pm 1.90$ & $11.47 \pm 1.07$ & $11.89 \pm 0.00$ & $11.65 \pm 1.06$ & 0.168 & 0.042 \\
\hline
\end{tabular}

Values are expressed as mean \pm standard deviation. Kruskal-Wallis analysis was used to compare continuous variables. 25(OH)D, serum 25-hydroxyvitamin D level; SPPB, short physical performance battery; NA, not available. 
Table 3. Regression analysis of physical performance according to 25(OH)D quartiles

\begin{tabular}{|c|c|c|c|c|c|c|}
\hline \multirow{2}{*}{ Variable } & \multicolumn{2}{|c|}{ Model 1} & \multicolumn{2}{|c|}{ Model 2} & \multicolumn{2}{|c|}{ Model 3} \\
\hline & $\beta$ & p-value & $\beta$ & p-value & $\beta$ & p-value \\
\hline \multicolumn{7}{|l|}{ Males $(n=63)$} \\
\hline Balance & NA & NA & NA & NA & NA & NA \\
\hline Walking & -0.037 & 0.455 & -0.039 & 0.435 & -0.036 & 0.496 \\
\hline Chair & -0.135 & 0.100 & -0.155 & 0.054 & -0.168 & 0.036 \\
\hline Total & -0.191 & 0.107 & -0.211 & 0.074 & -0.220 & 0.073 \\
\hline \multicolumn{7}{|c|}{ Females $(n=69)$} \\
\hline Balance & 0.018 & 0.542 & 0.012 & 0.680 & 0.014 & 0.626 \\
\hline Walking & 0.042 & 0.406 & 0.032 & 0.516 & 0.026 & 0.615 \\
\hline Chair & 0.213 & 0.018 & 0.187 & 0.032 & 0.176 & 0.046 \\
\hline Total & 0.273 & 0.042 & 0.231 & 0.073 & 0.216 & 0.095 \\
\hline
\end{tabular}

In Model 1, linear regression analysis was performed with serum 25(OH)D as an independent variable and each SPPB score as a dependent variable. Model 2 was adjusted for age. Model 3 was adjusted for the variables in Model 2 plus smoking, alcohol, physical activity, body mass index, and comorbidity.

25(OH)D, serum 25-hydroxyvitamin D level; SPPB, short physical performance battery; NA, not available.

\section{DISCUSSION}

The results of this study showed that vitamin D insufficiency was prevalent among community-dwelling older individuals in Korea. Most of the subjects in this study (77.3\%) had 25(OH)D levels below $30 \mathrm{ng} / \mathrm{mL}$. These results are similar to those reported by a previous study in Korea showing that 79.3\% of males and $75.3 \%$ of females had vitamin D levels below $30 \mathrm{ng} / \mathrm{mL} .^{33)}$ However, previous studies suggest that the prevalence of inadequate vitamin D levels in other countries is not as high as that in Korea. For example, the prevalence of vitamin D deficiency in Japan was much lower, at only around $5 \%$ of active older persons. In the present study, 36.51\% of males and 50.72\% of females had vitamin D levels below $20 \mathrm{ng} / \mathrm{mL}$; however, a recent study of Chinese individuals aged 60 years or older also reported that $34.1 \%$ of males and $44.0 \%$ of females had inadequate vitamin D levels $(<20 \mathrm{ng} / \mathrm{mL}) .{ }^{34)}$ Furthermore, countries with low latitudes, such as Australia, Indonesia, and Brazil, also have a lower prevalence of vitamin D deficiency. ${ }^{13)}$ These differences might be due to the differences in age, ethnicity, lifestyle, diets fortified with vitamin $\mathrm{D}$, or geographical difference leading to differences in sun exposure. ${ }^{35,36}$

We observed an association between low serum 25(OH) $\mathrm{D}$ levels and the chair stand scores in both sexes after adjustment for confounding factors (Model 3). However, there was no significant association with the other two parameters of the SPPB. There are two possible explanations for the discrepancy among SPPB parameters. First, the chair stand test requires more proximal lower extremity muscle strength. ${ }^{37)}$ Second, the differences may be due to the characteristics of the study population. This study only included relatively healthy older adults who went outdoors regularly and lived independently with or without the use of walking aids.

In this study, vitamin D insufficiency affected males and females differently. This difference between males and females could be explained by sex hormones and VDR gene polymorphisms. Sex hormones play a role in regulating vitamin D and calcium homeostasis. Estrogen regulates VDR expression and 1a-hydroxylase activity, while testosterone stimulates the expression of intestinal calcium channels. ${ }^{38,39)}$ The fact that males tend to be more physically active than females might also influence the results. In the present study, 30.2\% of males but only $18.8 \%$ of females exercised for more than 150 minutes per week. Females with poor SPPB scores who spend little time outdoors are likely to have low 25(OH)D levels, which is consistent with results of previous studies. ${ }^{40,411}$

The other possible explanations for why SPPB scores other than the chair stand score were not to related to vitamin D levels include age-related reductions in VDR expression. ${ }^{14)}$ Low vitamin D levels are common in older adults and long-term vitamin $\mathrm{D}$ deficiency might lead to the down-regulation of VDR. ${ }^{27)}$ In addition, VDR polymorphisms may also result in variable susceptibility to agerelated reductions in muscle performance. ${ }^{31)}$ Although we used a validated tool (SPPB) to evaluate physical performance, it may not fully reflect the overall physical performance.

The findings presented in this study are consistent with those of previous cross-sectional studies. For example, recent studies reported that higher 25(OH)D levels are associated with better musculoskeletal function in the lower extremities. ${ }^{42)}$ In the present study, the fully-adjusted model revealed a positive association between serum 25(OH) D levels and the chair stand scores in both sexes. Lower extremity function is considered an important factor for musculoskeletal health in older adults, as improved muscle strength can prevent disability, falls, and fractures. ${ }^{18)}$ Our results provide additional evidence that $25(\mathrm{OH}) \mathrm{D}$ is associated with physical function.

This study has several limitations. The first is a possible selection bias due to the recruitment of subjects who were in good physical condition. Our use of volunteers might 
mean that we inadvertently enrolled a relatively healthy older population because they were not inpatients or nursing home residents. Also, we excluded people who are not able to walk 10 m independently. Second, this was a crosssectional study; as such, we cannot determine cause-effect relationships, which may be bidirectional. Low vitamin D levels may cause poor physical performance. Conversely, physically active subjects are likely to spend time outdoors and longer sunlight exposure leads to higher endogenous vitamin D synthesis than that in sedentary subjects. Finally, the small sample size meant that the study lacked sufficient power to discriminate between subgroups. Individuals with lower 25(OH)D levels might be reasonably expected to have a more severe impairment with respect to SPPB; however, we could not perform subgroup analysis of patients with respect to SPPB scores or different vitamin D levels.

This study also has several strengths. First, we used the SPPB test, which is a standardized and validated test for use in older populations that is a good predictor of falls, inflammatory reactions, and declining function; it also assesses maximum functional outcome rather than lower extremity disability. ${ }^{18,25)}$ Second, the high prevalence of $25(\mathrm{OH}) \mathrm{D}$ deficiency in this population made it a good sample in which to investigate the relationship between vitamin D and physical performance. ${ }^{38)}$ All of the subjects lived in an urban area in Korea, which is likely related to a sun-shy lifestyle and poor exposure to sunlight when compared to sunnier countries such as Australia, Indonesia, and Brazil. ${ }^{13)}$ Third, we used an easily replicable model. The strategy used in this study only added two tests (serum $25(\mathrm{OH}) \mathrm{D}$ levels and SPPB) to the routine laboratory tests commonly used in outpatient clinics or during regular check-ups. Therefore, it is easy to implement, even in a primary clinic or in hospital-based care, because training or specialized equipment are not required.

Lower serum 25(OH)D levels were associated with poor chair stand scores in ambulatory Koreans aged over 60 years, independent of age, BMI, smoking status, physical activity, and comorbidities. There was no association between serum 25(OH)D levels and the other SPPB scores. Serum 25(OH)D may be an important indicator for various health outcomes, including physical performance, in older adults. Further longitudinal studies are needed to clarify the effects of vitamin D supplementation with respect to improving skeletal muscle function and reducing falls and fractures. In addition, vitamin D levels in older adults should be monitored. Patients should be educated about the importance of sun exposure along with a healthy lifestyle. Primary prevention of vitamin D deficiency is needed to improve musculoskeletal health in later life. Public health strategies should target this population.

\section{CONFLICTS OF INTEREST DISCLOSURES}

The researchers claim no conflicts of interest.

\section{ACKNOWLEDGMENTS}

This work was funded by Biomedical Research Institute grant, Kyungpook National University Hospital (2016NEW-06).

\section{REFERENCES}

1. Chapuy MC, Preziosi P, Maamer M, Arnaud S, Galan P, Hercberg $\mathrm{S}$, et al. Prevalence of vitamin D insufficiency in an adult normal population. Osteoporos Int 1997;7:439-43.

2. Close GL, Leckey J, Patterson M, Bradley W, Owens DJ, Fraser WD, et al. The effects of vitamin $\mathrm{D}(3)$ supplementation on serum total 25[OH]D concentration and physical performance: a randomised dose-response study. Br J Sports Med 2013;47:692-6.

3. Lamberg-Allardt C, Brustad M, Meyer HE, Steingrimsdottir L. Vitamin D - a systematic literature review for the 5th edition of the Nordic Nutrition Recommendations. Food Nutr Res 2013;57:22671.

4. Mithal A, Wahl DA, Bonjour JP, Burckhardt P, Dawson-Hughes B, Eisman JA, et al. Global vitamin D status and determinants of hypovitaminosis D. Osteoporos Int 2009;20:1807-20.

5. Granic A, Hill TR, Davies K, Jagger C, Adamson A, Siervo M, et al. Vitamin D status, muscle strength and physical performance decline in very old adults: a prospective study. Nutrients 2017;9:E379.

6. Bischoff HA, Stähelin HB, Dick W, Akos R, Knecht M, Salis C, et al. Effects of vitamin D and calcium supplementation on falls: a randomized controlled trial. J Bone Miner Res 2003;18:343-51.

7. Glerup H, Mikkelsen K, Poulsen L, Hass E, Overbeck S, Andersen H, et al. Hypovitaminosis D myopathy without biochemical signs of osteomalacic bone involvement. Calcif Tissue Int 2000;66:419-24.

8. Bischoff-Ferrari HA, Dawson-Hughes B, Staehelin HB, Orav JE, Stuck AE, Theiler R, et al. Fall prevention with supplemental and active forms of vitamin D: a meta-analysis of randomised controlled trials. BMJ 2009;339:b3692.

9. Baczynski R, Massry SG, Magott M, el-Belbessi S, Kohan R, Brautbar N. Effect of parathyroid hormone on energy metabolism of skeletal muscle. Kidney Int 1985;28:722-7.

10. Lips P. Vitamin D deficiency and secondary hyperparathyroidism in the elderly: consequences for bone loss and fractures and therapeutic implications. Endocr Rev 2001;22:477-501.

11. Chapuy MC, Arlot ME, Duboeuf F, Brun J, Crouzet B, Arnaud S, et al. Vitamin D3 and calcium to prevent hip fractures in elderly women. N Engl J Med 1992;327:1637-42.

12. Choi HS, Oh HJ, Choi H, Choi WH, Kim JG, Kim KM, et al. Vitamin D insufficiency in Korea--a greater threat to younger generation: the Korea National Health and Nutrition Examination Survey (KNHANES) 2008. J Clin Endocrinol Metab 2011;96:643-51.

13. Diekmann R, Winning K, Bauer JM, Uter W, Stehle P, Lesser S, et al. Vitamin D status and physical function in nursing home residents: a 1-year observational study. Z Gerontol Geriatr 2013;46:403-9.

14. Bischoff-Ferrari HA, Borchers M, Gudat F, Dürmüller U, Stähelin HB, Dick W. Vitamin D receptor expression in human muscle tissue decreases with age. J Bone Miner Res 2004;19:265-9. 
15. Grimby G, Saltin B. The ageing muscle. Clin Physiol 1983;3:20918.

16. Laforest S, St-Pierre DM, Cyr J, Gayton D. Effects of age and regular exercise on muscle strength and endurance. Eur J Appl Physiol Occup Physiol 1990;60:104-11.

17. Bischoff HA, Stahelin HB, Urscheler N, Ehrsam R, Vonthein R, Perrig-Chiello $\mathrm{P}$, et al. Muscle strength in the elderly: its relation to vitamin D metabolites. Arch Phys Med Rehabil 1999;80:54-8.

18. Guralnik JM, Branch LG, Cummings SR, Curb JD. Physical performance measures in aging research. J Gerontol 1989;44:M141-6.

19. Ning Z, Song S, Miao L, Zhang P, Wang X, Liu J, et al. High prevalence of vitamin D deficiency in urban health checkup population. Clin Nutr 2016;35:859-63.

20. Kim MK, Lee WY, Kang JH, Kang JH, Kim BT, Kim SM, et al. 2014 clinical practice guidelines for overweight and obesity in Korea. Endocrinol Metab (Seoul) 2014;29:405-9.

21. Centers for Disease Control and Prevention (CDC). State-specific secondhand smoke exposure and current cigarette smoking among adults - United States, 2008. MMWR Morb Mortal Wkly Rep 2009;58:1232-5.

22. Centers for Disease Control and Prevention. Vital signs: alcoholimpaired driving among adults--United States, 2010. MMWR Morb Mortal Wkly Rep 2011;60:1351-6.

23. Ainsworth BE, Haskell WL, Herrmann SD, Meckes N, Bassett DR Jr, Tudor-Locke C, et al. 2011 Compendium of physical activities: a second update of codes and MET values. Med Sci Sports Exerc 2011;43:1575-81.

24. Jung HY, Kim MY, Kim JM, Kim DW, Kim CB. Vitamin D deficiency in nursing home elderly in Korea. J Korean Geriatr Soc 2016;20:102-7.

25. Bjerk SM, Edgington BD, Rector TS, Kunisaki KM. Supplemental vitamin D and physical performance in COPD: a pilot randomized trial. Int J Chron Obstruct Pulmon Dis 2013;8:97-104.

26. de Koning EJ, van Schoor NM, Penninx BW, Elders PJ, Heijboer AC, Smit JH, et al. Vitamin D supplementation to prevent depression and poor physical function in older adults: study protocol of the D-vitaal study, a randomized placebo-controlled clinical trial. BMC Geriatr 2015;15:151.

27. Johnson LK, Hofsø D, Aasheim ET, Tanbo T, Holven KB, Andersen LF, et al. Impact of gender on vitamin D deficiency in morbidly obese patients: a cross-sectional study. Eur J Clin Nutr 2012;66:83-90.

28. Lim JS, Kim KM, Rhee Y, Lim SK. Gender-dependent skeletal effects of vitamin D deficiency in a younger generation. J Clin Endocrinol Metab 2012;97:1995-2004.

29. Sanghera DK, Sapkota BR, Aston CE, Blackett PR. Vitamin D status, gender differences, and cardiometabolic health disparities. Ann Nutr Metab 2017;70:79-87.

30. Houston DK, Cesari M, Ferrucci L, Cherubini A, Maggio D, Bartali
B, et al. Association between vitamin D status and physical performance: the InCHIANTI study. J Gerontol A Biol Sci Med Sci 2007;62:440-6.

31. Matheï C, Van Pottelbergh G, Vaes B, Adriaensen W, Gruson D, Degryse JM. No relation between vitamin D status and physical performance in the oldest old: results from the Belfrail study. Age Ageing 2013;42:186-90.

32. Wicherts IS, van Schoor NM, Boeke AJ, Visser M, Deeg DJ, Smit $\mathrm{J}$, et al. Vitamin D status predicts physical performance and its decline in older persons. J Clin Endocrinol Metab 2007;92:2058-65.

33. Nah EH, Kim S, Cho HI. Vitamin D levels and prevalence of vitamin D deficiency associated with sex, age, region, and season in Koreans. Lab Med Online 2015;5:84.

34. Chen J, Yun C, He Y, Piao J, Yang L, Yang X. Vitamin D status among the elderly Chinese population: a cross-sectional analysis of the 2010-2013 China National Nutrition and Health Survey (CNNHS). Nutr J 2017;16:3.

35. Nakamura K. Vitamin D insufficiency in Japanese populations: from the viewpoint of the prevention of osteoporosis. J Bone Miner Metab 2006;24:1-6.

36. Rinaldi I, Setiati S, Oemardi M, Aries W, Tamin TZ. Correlation between serum vitamin D (25(OH)D) concentration and quadriceps femoris muscle strength in Indonesian elderly women living in three nursing homes. Acta Med Indones 2007;39:107-11.

37. Lagari V, Gómez-Marín O, Levis S. The role of vitamin D in improving physical performance in the elderly. J Bone Miner Res 2013;28:2194-201.

38. Boyé ND, Oudshoorn C, van der Velde N, van Lieshout EM, de Vries OJ, Lips P, et al. Vitamin D and physical performance in older men and women visiting the emergency department because of a fall: data from the improving medication prescribing to reduce risk of falls (IMPROveFALL) study. J Am Geriatr Soc 2013;61:1948-52.

39. Grundberg E, Brändström H, Ribom EL, Ljunggren 0, Mallmin H, Kindmark A. Genetic variation in the human vitamin D receptor is associated with muscle strength, fat mass and body weight in Swedish women. Eur J Endocrinol 2004;150:323-8.

40. Dam TT, von Mühlen D, Barrett-Connor EL. Sex-specific association of serum vitamin D levels with physical function in older adults. Osteoporos Int 2009;20:751-60.

41. Gerdhem P, Ringsberg KA, Obrant KJ, Akesson K. Association between 25-hydroxy vitamin D levels, physical activity, muscle strength and fractures in the prospective population-based OPRA Study of Elderly Women. Osteoporos Int 2005;16:1425-31.

42. Bischoff-Ferrari HA, Dietrich T, Orav EJ, Hu FB, Zhang Y, Karlson EW, et al. Higher 25-hydroxyvitamin D concentrations are associated with better lower-extremity function in both active and inactive persons aged > or =60 y. Am J Clin Nutr 2004;80:752-8. 